\title{
Gestão do conhecimento como um elemento chave para a geração de inteligência competitiva organizacional
}

Knowledge management as a key element for the organizational competitive intelligence

generation

\author{
Fábio Aquino da Silva ${ }^{1}$ \\ Geisa Meirelles Drumond² \\ Haydée Maria Correia da Silveira Batista ${ }^{3}$ \\ Marcelo Jasmim Meiriño ${ }^{4}$ \\ Mirian Picinini Méxas ${ }^{5}$
}

\begin{abstract}
Resumo
A Gestão do Conhecimento na esfera organizacional traz novas perspectivas para o negócio, pois gera valor para os processos fundamentais para o desenvolvimento de produtos e serviços. Dentro deste contexto, este estudo tem como objetivo analisar, numa perspectiva polissêmica, os fundamentos, características e aplicações da gestão do conhecimento no desenvolvimento organizacional e demonstrar como através da inteligência competitiva, construída sobre conhecimento e competência informacional, é possível obter bons resultados e melhorar o posicionamento no mercado, utilizando o modelo proposto de geração de inteligência competitiva organizacional. Com base na análise da literatura, verificou-se que a percepção do alinhamento entre Gestão do Conhecimento e objetivos organizacionais é importante para garantir o ajuste de ações com os objetivos estratégicos da empresa, constituindo, portanto, um diferencial competitivo nas organizações. Além disso, a Gestão do Conhecimento sistematizada pela ferramenta PDCA, implementada em ações e processos contínuos, traz bons resultados, como o envolvimento do nível estratégico da organização que, através do uso da tecnologia como ferramenta, cria uma plataforma para as comunidades de práticas virtuais.
\end{abstract}

Palavras-chave: Gestão do Conhecimento, Inteligência competitiva, Competência informacional.

\begin{abstract}
Knowledge Management in the organizational sphere brings new perspectives to the business, since it generates value for the processes that is fundamental for the development of products and services. Within this context, this study aims to analyze, in a polysemy perspective, the fundamentals, characteristics and applications of knowledge management in organizational development and demonstrate how through competitive intelligence, built on knowledge and informational competence, it is possible to achieve good results and improve the positioning in the market, using the proposed model of organizational competitive intelligence generation. Based on the literature analysis, it was verified that perception of the alignment between Knowledge Management and organizational objectives is important to ensure adjustment of actions with the strategic objectives of the company constituting, therefore, a competitive differential in organizations. In addition, Knowledge Management systematized by the PDCA tool, implemented in continuous actions and processes, brings good results, such as the involvement of the organization's strategic level, which, with technology as a tool, creates a platform for the communities of virtual practices.
\end{abstract}

Keywords: Knowledge Management, Competitive intelligence, Informational competence.

\footnotetext{
${ }^{1}$ Doutorando em Sistemas de Gestão Sustentável. UFF/ Brasil. E-mail: faquin98@gmail.com

${ }^{2}$ Doutoranda em Sistemas de Gestão Sustentáveis, UFF/ Brasil. E-mail: meirellesdrumond@gmail.com

${ }^{3}$ Doutoranda em Engenharia Civil.UFRJ/Brasil - Bolsista de Doutorado pela CNPq - E-mail: haydeemcsb@gmail.com

${ }^{4}$ Professor do Mestrado em Sistemas de Gestão e do Doutorado em Sistemas de Gestão Sustentáveis. UFF/

Brasil. E-mail: marcelojm@id.uff.br

${ }^{5}$ Professora do Mestrado em Sistemas de Gestão e do Doutorado em Sistemas de Gestão Sustentáveis. UFF/

Brasil. E-mail: mirian mexas@id.uff.br
} 


\section{INTRODUÇÃO}

O conhecimento é objeto de muitos estudos sendo reconhecido em várias perspectivas como um ativo fundamental para o desenvolvimento da economia e das organizações (DRUCKER, 1968). Com o crescimento das pesquisas sobre a Gestão do Conhecimento (GC) no âmbito organizacional, novas perspectivas para os negócios surgiram, uma vez que geram valor para os processos, que é fundamental para o desenvolvimento de produtos e serviços (AL -EMRAN et al., 2018; GAVIRIA-MARIN; MERIGÓ; BAIER-FUENTES, 2018).

Aliada à gestão do conhecimento, o conceito de inteligência competitiva organizacional (ICO) engloba o monitoramento de informações internas e externas para a tomada de decisões em diferentes níveis estratégicos (WANG, 2014). Nesse sentido, um fator importante para obtenção e criação de conhecimento estratégico refere-se a um conjunto de competências organizacionais, visando o fortalecimento e o contínuo desenvolvimento da inteligência competitiva (TRIEU, 2017).

Para entender melhor como esses conceitos se articulam, buscaram-se, na literatura, embasamentos teóricos e empíricos para nortear a construção desse estudo, visando uma compreensão mais ampla da contribuição da gestão do conhecimento na construção de vantagens nas organizações. Além disso, ressalta-se a necessidade de se discutir sobre modelos de maturidade em busca da melhoria dos processos relacionados à GC, para facilitar a sua implantação nas organizações, identificando barreiras e oportunidades para o atingimento de metas e objetivos.

Com base nessas considerações, o objetivo deste estudo é analisar em uma perspectiva polissêmica, os fundamentos, características e aplicações da gestão do conhecimento no desenvolvimento organizacional e demonstrar como por meio da inteligência competitiva, construída sobre o conhecimento e a competência informacional, é possível alcançar bons resultados e melhorar o posicionamento no mercado.

\section{REVISÃO DE LITERATURA}

Nesta seção, foram abordados os conceitos de gestão do conhecimento, inteligência competitiva organizacional, bem como a influência da gestão da informação, gestão do conhecimento e tecnologia da informação na inteligência competitiva organizacional.

\subsection{FUNDAMENTOS DA GESTÃO DO CONHECIMENTO}

A gestão do conhecimento pode ser definida como a transformação do conhecimento individual em conhecimento organizacional para todos os envolvidos com a empresa, o que influencia a organização a manter sólidos mecanismos que orientam a formatação do sistema de práticas de GC existentes (LOON, 2019). Com isso, o conhecimento pessoal tem destaque nas organizações como a atividade central da empresa criadora de conhecimento (WILLERDING; KRAUSE; LAPOLLI, 2016).

A preocupação das organizações com os seus valiosos conhecimentos e informações é fundamentada pelas estruturas intelectuais de conhecimentos que detêm em sua disposição a movimentação do conhecimento tácito dos seus membros e a disseminação do conhecimento explícito (AHMAD; BOSUA; SCHEEPERS, 2014; MARCHIORI; FRANCO, 2019). 
Assim, o gerenciamento do conhecimento proporciona o crescimento do capital intelectual da organização, que contribui na construção do valor dos produtos e serviços, o que valoriza os recursos humanos empregados na empresa, proporcionando, assim, um diferencial competitivo.

O processo de inteligência organizacional (IO) apresenta uma relação com o processo de construção e gestão do conhecimento, que pode ser percebido de uma maneira mais nítida no momento da interpretação e análise das informações prospectadas, contribuindo para a construção de conhecimento (JORGE; SUTTON, 2016).

Deste modo, a cultura organizacional é uma mediadora, sendo influenciada pelas atitudes e comportamentos dos membros organizacionais que passam a agir e reagir às informações disponibilizadas para o desempenho das tarefas que compõem o trabalho com um novo conhecimento para os ambientes organizacionais (LOON, 2019).

Além disso, o desenvolvimento do processo de inteligência organizacional - 10 precisa ser alimentado pelo processamento cognitivo dos sujeitos organizacionais com o objetivo de gerar a novidade. Esta ação deve formatar uma perspectiva de sustentabilidade corporativa que contribuirá com a interpretação de determinada situação, favorecendo o aspecto fundamental no processo de construção do 'novo' conhecimento que será oriundo da socialização e/ou compartilhamento do conhecimento em um contexto organizacional (GREWATSCH; KLEINDIENST 2018).

Assim, o conhecimento passa por uma transformação sendo modificado pela interpretação cognitiva dos demais sujeitos organizacionais, contribuindo para a construção dos novos conhecimentos. Sendo assim, serão futuramente definidos como conhecimento não explicitado, chamado de conhecimento tácito, ou seja, com ênfase no capital intelectual e sua ação restrita à cultura e comunicação organizacional que pode ser mantido como uma vantagem competitiva (OLAISEN; REVANG, 2018).

$\mathrm{Na}$ atuação da GC, em regra, ocorre a dinâmica criação dos processos de aquisição, armazenamento, aplicação, compartilhamento ou transferência do conhecimento, que tem início na geração de ideias, a qual utiliza o aprendizado na avaliação de conhecimentos, experiências e especializações das organizações (SIMANCA; MONTOYA; BERNAL, 2016), embora a ênfase desta ação também favoreça que as pessoas encontrem condições e um ambiente favorável, onde é possível valorizar as experiências passadas que formam a estrutura de referência para os processos de aprendizados futuros (OLIVEIRA; SAUER, 2016).

A palavra ba tem como tradução do japonês "um tempo específico e espaço" o que não é necessariamente um espaço físico, pode ser virtual e mental que por ser um termo relativamente novo ainda não tem definição acadêmica estabelecida (ASHRAFI et al., 2019; NONAKA; TOYAMA; KONNO, 2000).

Assim, no planejamento organizacional, o termo ba pode ser caracterizado como um contexto ou "campo" no qual o conhecimento é compartilhado, criado e utilizado, o que envolve trocas humanas, com difícil verbalização, mas que, se bem exploradas, podem reduzir as dificuldades que impedem as mudanças organizacionais (PARK; GABBARD, 2018; ZHANG; CHENG, 2015). A Figura 1 mostra um contexto compartilhado em movimento. 


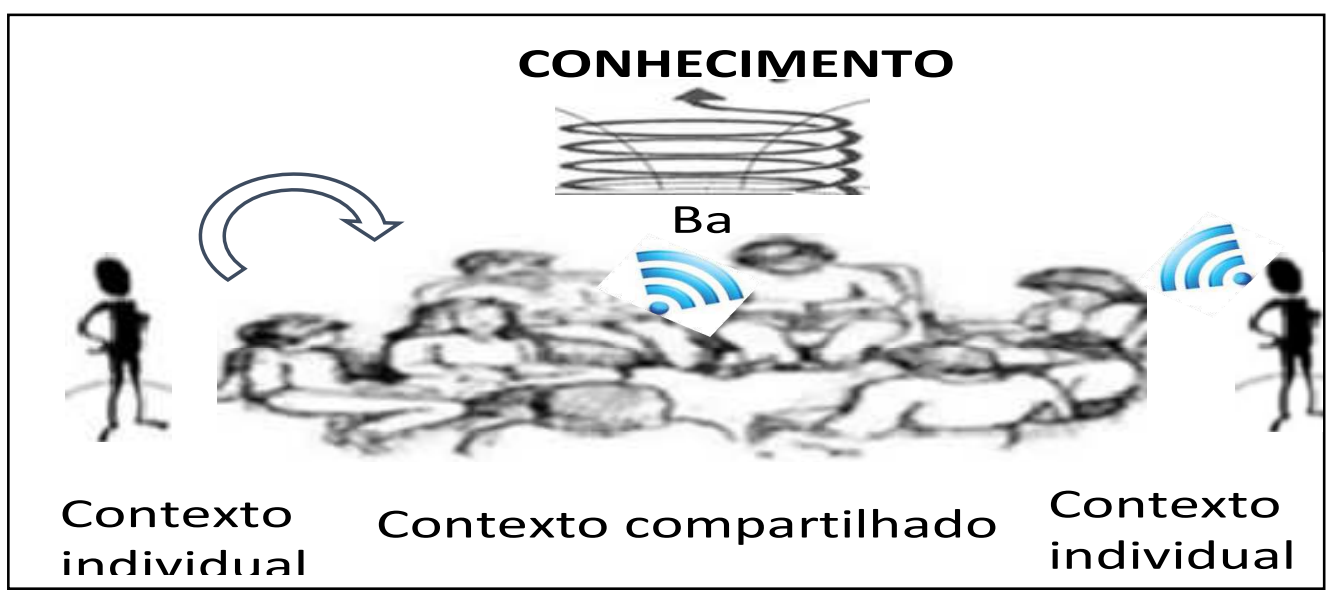

Figura 1 - Ba: contribuindo para a produção do conhecimento Fonte: Elaborada pelos autores (2019)

A concepção do ba, como ilustrado na Figura 1, contribui com aspectos teóricos e empíricos da GC e com a possibilidade de uso da inteligência como ferramenta que irá apoiar a organização e que tem como característica a valorização do conhecimento tácito, ou seja, a importância que deve ser atribuída, conforme a realidade contextual, conscientização, ao diálogo e à positividade presente na colaboração humana (SERGEEVA; ANDREEVA, 2016).

\subsection{INTELIGÊNCIA COMPETITIVA ORGANIZACIONAL}

O conceito e a prática de inteligência competitiva organizacional (ICO) apresentam duas vertentes com algumas diferenças. A primeira define inteligência competitiva como um programa com base em uma sistemática ética para reunir e analisar a informação externa, principalmente por ser uma variável que representa a organização no mercado, por meio de um entendimento maior, mas inequívocamente ético, dos envolvidos na competição e do seu ambiente competitivo (ŠTEFÁNIKOVÁ; MASÀROVÀ 2014). Já na segunda definição, a compreensão aborda a importância do acompanhamento da concorrência de mercado competitivo por meio da prospecção e do monitoramento da concorrência e do mercado competitivo, que é a principal ação estratégica para a sobrevivência organizacional, que é baseada na previsão das tendências de movimentar-se mais rápido que a concorrência (TEIXEIRA; VALENTIM, 2016).

No Brasil, a definição da Associação Brasileira dos Analistas de Inteligência Competitiva - ABRAIC justifica a ICO como um processo que abrange todos os níveis organizacionais, sendo informacional e proativo para identificar forças de reação ao negócio, com o objetivo de reduzir o risco e contribuir para a tomada de decisão, protegendo sempre o conhecimento gerado. Desta forma, a inteligência competitiva é formalizada nas informações internas e externas, para subsidiar as decisões organizacionais, o que irá agregar na análise de dados, de modo a utilizar o know-how e o conhecimento individual e organizacional (KHAN, 2019).

A inteligência competitiva (IC) proporciona vantagens à organização, quando o processo de busca, o compartilhamento e o uso de informações se tornam uma constante, estabelecendo uma dinâmica em relação à rapidez, tempo, resultado e impacto no mercado, 
bem como a antecipação às ameaças, e aproveitando as oportunidades de maneira eficiente. A relação existente entre os elementos demanda à gestão do conhecimento um alinhamento informacional entre as demandas internas e externas para facilitar a análise da inteligência produzida (SANTOS; SANTOS; BELLUZZO, 2016).

Dessa forma, a organização pode desenvolver um processo de aprendizagem baseado em competição e em informação, que também fornece insights práticos sobre o uso instrumental e intencional de ferramentas, como arte e design, como mecanismo de tradução do conhecimento que facilita e potencializa o fluxo de comunicação e colaboração, que terá impacto direto nas estratégias de negócios de curto e longo prazo (SIMEONE; SECUNDO; SCHIUMA, 2018)

O foco dessa ação está no alinhamento entre as linhas de pesquisa sobre a interrelação entre inteligência competitiva, informação, conhecimento e know-how com conhecimento individual (KHAN, 2019).

\subsection{OS PRINCIPAIS FATORES QUE INFLUENCIAM A INTELIGÊNCIA COMPETITIVA ORGANIZACIONAL}

A persistência organizacional para a busca, compartilhamento e uso da informação estabelece uma dinâmica entre a gestão do conhecimento e outras áreas da organização e, quando integrada, favorece a transferência de conhecimento através da criação de artefatos de design em vários níveis de precisão que respondem com uma busca mais rápida a resultados e impactos no mercado, além de antecipar ameaças e aproveitar oportunidades de forma eficiente (SIMEONE; SECUNDO; SCHIUMA, 2018). A relação entre os elementos provoca a gestão do conhecimento com um alinhamento informacional entre as demandas internas e externas para facilitar a análise da inteligência produzida (SANTOS; SANTOS; BELLUZZO, 2016).

As organizações têm, em regra, como característica o uso de três fatores que se integram para assegurar o sucesso do processo de ICO, são eles: o fator cultural, o fator estrutural e o fator comportamental (ALI et al., 2019; CHIU; YANG, 2019; MILLER, 2002). Desta forma, o fator cultural está relacionado aos encadeamentos necessários para a criação do conhecimento, sendo a origem da demanda as vontades cognitivas e as angústias dos sujeitos organizacionais pela ausência da informação, de suas emoções antecipadas, até o estimulo para encontrar a informação (DERVIN, 1992; PIÇARRA; GIGER, 2018).

O fator estrutural está relacionado com o ambiente onde a informação fica disponível para o uso das pessoas, favorecendo a partilha do grupo, visando proporcionar a construção de soluções para os problemas, conforme a informação recebida. Deste modo, o desenvolvimento de ideias acontece conforme o perfil dos grupos envolvidos na busca das informações para o problema (CHEN; HSINHUI; KING, 2018).

O terceiro fator que deve ser usado pela organização é o comportamental, que consiste nas ações de busca da informação e a efetiva coleta dos dados, observando a ação da dimensão emocional que influencia a clara compreensão da necessidade de informação que é traduzida em perguntas ou tópicos para orientar a busca (HELLE et al. 2018). A Figura 2 representa a influência do uso dos fatores da ICO. 


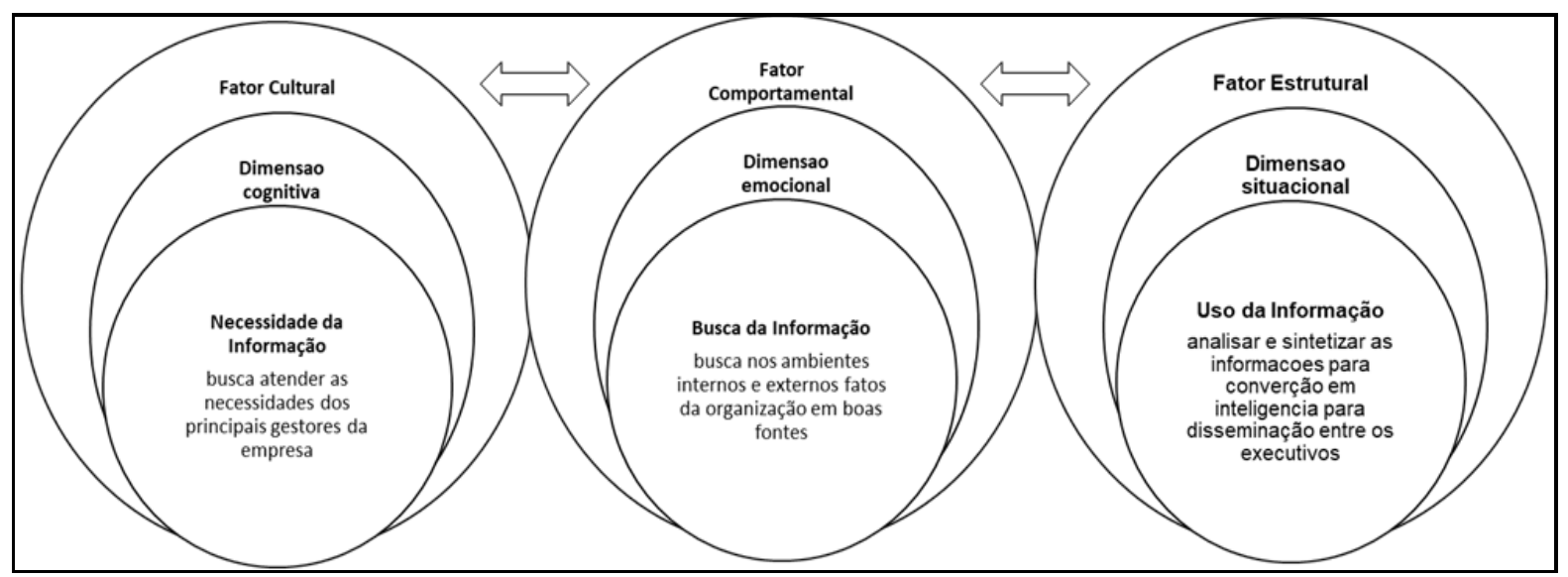

Figura 2 - Fatores de influência da ICO

Fonte: Elaborada pelos autores com base nos estudos de Miller (2002) e Choo (2006)

A ICO representa uma ferramenta que favorece o alinhamento das informações e a construção de uma combinação de síntese teórica com processos inter-relacionados que poderá orientar, quando sistematizada, a análise dos critérios de respaldo à tomada de decisão mantendo o foco no cliente (CEPEDA-CARRION et al. 2017). Assim, a ICO representa o apoio para a alta gerência que poderá com maior facilidade interpretar as informações coletadas e planejar a estratégia de comunicação organizacional necessária para evitar que ocorram ruídos ao longo do processo.

Além disso, a organização pode desenvolver um processo de aprendizado com foco na competição, tendo como base as informações que irão manter a sustentabilidade das vantagens e impactar diretamente as estratégias empresariais de curto e longo prazo (GONZÁLEZ-LOUREIRO, 2015; VILA ALONSO; SCHIUMA, 2015). O objetivo desta ação é alinhar as linhas de investigação sobre a inter-relação entre inteligência competitiva, informação e conhecimento, e know-how com o conhecimento individual.

\subsection{INFLUÊNCIA DA GESTÃO DA INFORMAÇÃO, DA GESTÃO DO CONHECIMENTO E DAS TECNOLOGIAS DA INFORMAÇÃO E COMUNICAÇÃO NA INTELIGÊNCIA COMPETITIVA ORGANIZACIONAL}

O processo de formação da ICO depende de algumas práticas que evidenciam a influência de um conjunto de tecnologias e processos que favorecem a criação e transferência do conhecimento de conceitos com muitas faces e complexos (BEKER et al. 2019). Desta forma, o fluxo sistematizado deve proporcionar uma constante retroalimentação ao processo com destaque para as atividades de monitoramento e prospecção das informações mais importantes para o contexto analisado. Com isso, a informação é agregada e armazenada, utilizando Tecnologias da Informação e Comunicação (TIC) para favorecer o meio de propagação do resultado da ação de inteligência que em uma ação futura irá alimentar novamente o sistema e a ICO (TEIXEIRA; VALENTIM 2016).

Deste modo, a sistematização deste processo caracteriza uma relação de dependência entre as TIC e a ICO. Segundo a literatura, trata-se de um Sistema de Inteligência Competitiva (SIC), por facilitar o alinhamento entre os ativos e a cultura necessária, por meio das etapas de identificar, capturar e analisar as informações já 
direcionadas para a tomada de decisão no nível estratégico da organização (EREVELLES; FUKAWA; SWAYNE, 2016; JAKLIČ; GRUBLJEŠIČ; POPOVIČ, 2018). Assim, ao aplicar a metodologia é importante observar que não são usados somente os elementos básicos de identificação da informação, mas técnicas e ferramentas são aplicadas para facilitar que sejam identificadas as informações mais importantes sobre a concorrência, como representado na Figura 3.

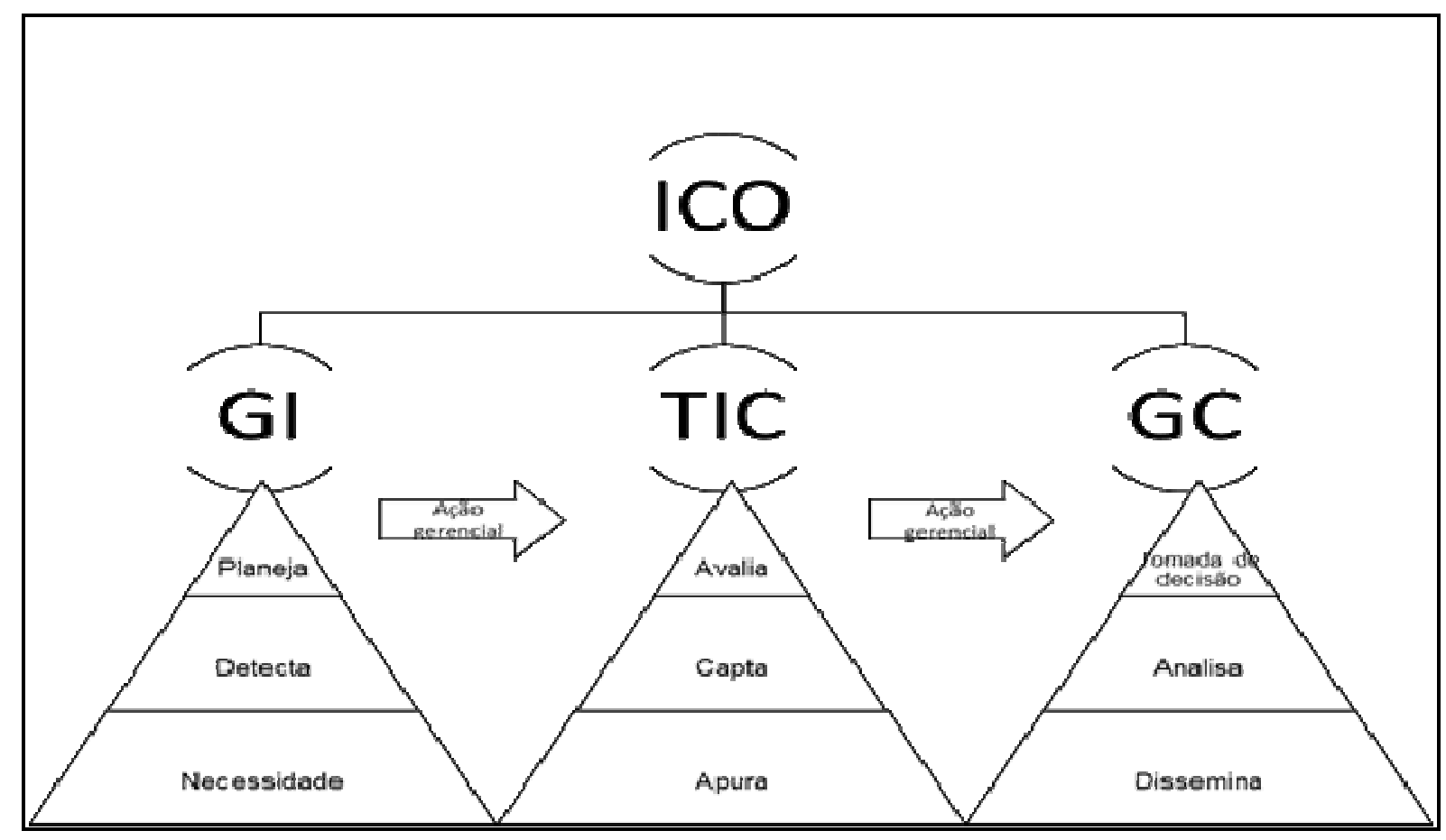

Figura 3 - Sistematização do processo da ICO Fonte: Elaborada pelos autores (2019)

A Figura 3 demonstra a importância das correlações existentes entre a gestão da Informação (GI), a gestão do conhecimento $(G C)$ e o uso de tecnologias de informação e comunicação (TIC) como fundamentos para a construção da geração de inteligência. Sendo assim, é observada a contribuição com o contexto organizacional e o uso da correlação entre as atividades de planejamento, avaliação e tomada de decisão que organizam e formatam o processo de entrega de valor em relação à criação de conhecimento frente às demandas, observando a necessidade de busca, o compartilhamento e uso de informação (BOžlČ; DIMOVSKI, 2019)

Em uma análise do conjunto apresentado na Figura 3 com ações cotidianas vivenciadas pelos sujeitos organizacionais que dependem desses elementos, é percebida a importância das etapas de prospecção e o monitoramento (TEIXEIRA; VALENTIM, 2016).

Assim, ocorre uma melhoria nos fluxos de coleta, organização, análise e implementação de mudanças. Já a competitividade organizacional é influenciada positivamente pela qualidade da informação estratégica gerada. 


\subsection{MODELO DE MATURIDADE}

O Modelo de maturidade das contribuições em estágios sucessivos em direção à melhoria contínua de processos é composto por cinco estágios de desenvolvimento, para ser implantado em uma empresa; estas etapas foram mapeadas e classificadas pela engenharia de software (DALKIR, 2013; KRAEMER et al., 2017). O modelo CLM - Configuration Lifecycle Management - propõe o gerenciamento do ciclo de vida dos modelos usados na produção da indústria e, por isso, pode ser replicado no uso da GC como uma ferramenta que favorece o compartilhamento do conhecimento e os dados sobre a operação com todas as áreas da organização (MYRODIA; RANDRUP; HVAM, 2019).

Assim como o modelo de GC, os resultados da utilização desses modelos são importantes tanto para os gestores organizacionais quanto para a comunidade acadêmica, por orientar sistematicamente os usuários e stakeholders, por avaliar o estado atual de uma organização, além de incluir fornecedores e outros stakeholders e a produção do processo de conhecimento.

A Figura 4 mostra os cinco níveis de maturidade organizacional descritos a seguir: as capacidades iniciais que fundamentam o primeiro nível são o estágio em que a estrutura e o escopo do plano de comunicação interno são definidos em conjunto com as equipes ou departamentos dedicados como parte da organização para apoiar a jornada CLM.

O segundo estágio é a definição do comportamento departamental por meio de papéis e responsabilidades das equipes usando linhas claras de responsabilidade para cada produto e fase.

O terceiro estágio é definido como a especialização interorganizacional que tem como característica a colaboração entre as organizações nas tarefas do CLM entre as partes internas e fornecedores envolvidos. No quarto estágio, a chamada expertise externa focada, onde a organização tem uma equipe ou departamento dedicado a envolver os interesses externos por meio das pratica de governança, monitoramento, ciclo de feedback, gestão de mudança.

O último estágio é a melhoria contínua onde qualquer alteração no CLM é participada aos interessados e simultaneamente disseminada para as partes internas e externas da organização e tem como foco a verificação de novas ideias, ao invés de uma análise qualitativa (KOOMEN, 2017). 


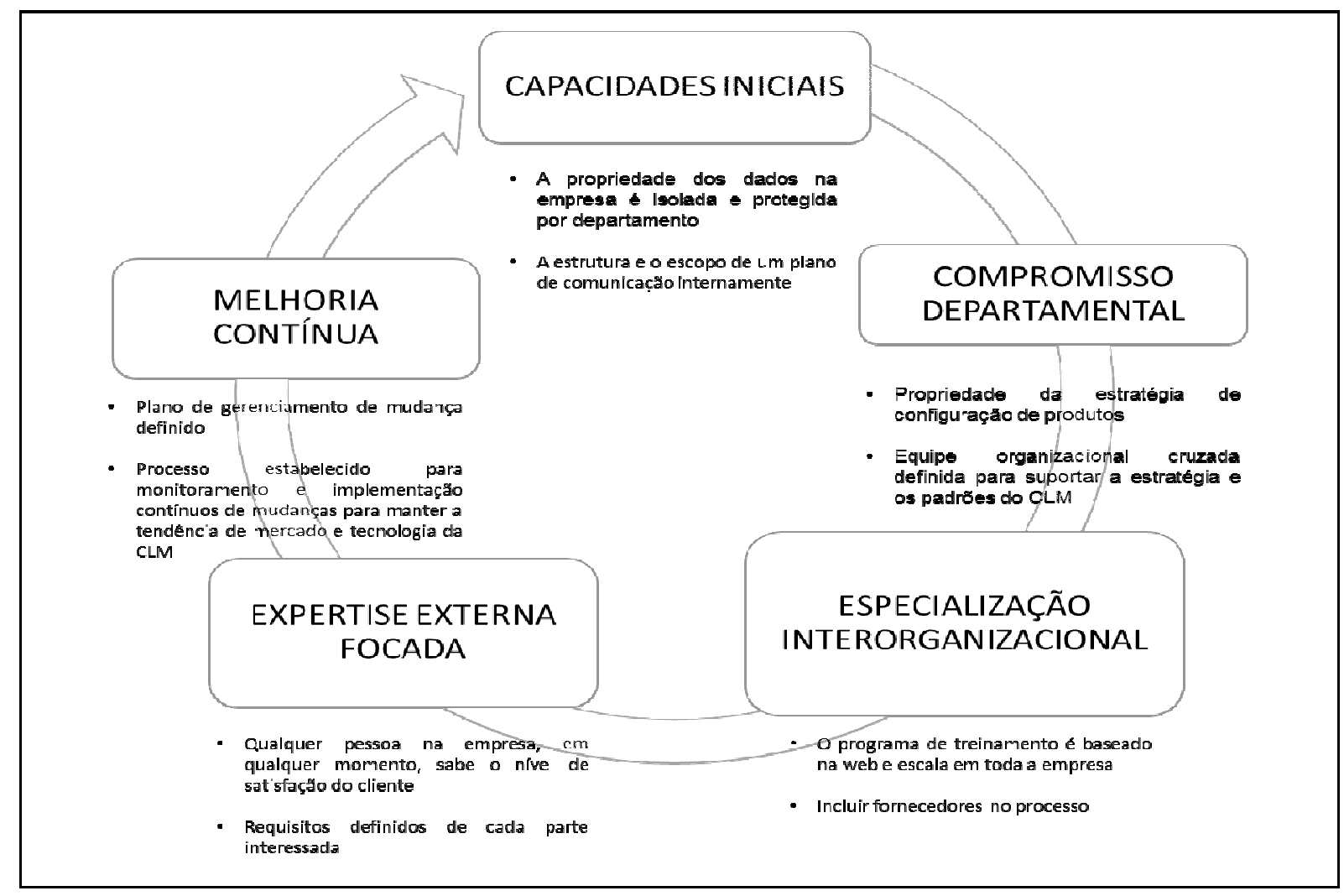

Figura 4 - Etapas do modelo CLM aplicado a organização Fonte: Elaborada pelos autores (2019)

Assim, os estágios interligados e sendo retroalimentados proporcionam o que a revisão de literatura apresenta como as condições necessárias para a verificação organizacional de alguns modelos de GC para atingir o nível de maturidade do processo, e parte deles sendo fundamentada pelo CLM, como uma ferramenta que faz a verificação do atingimento de metas e objetivos (KÄRKKÄINEN et al., 2014).

Sendo assim, a importância do uso de modelos de maturidade dedicados à GC é fundamentada em quatro aspectos: o primeiro deles é a implementação bem-sucedida das iniciativas de GC por exigir uma abordagem sistematizada e estruturada para desenvolver a medição, que proporcionará a melhoria contínua dos processos organizacionais diretamente relacionados (KHADDAJ; SROUR, 2016). Já em um segundo aspecto, os modelos de maturidade têm uma característica eficaz para facilitar a GC na organização. Em terceiro lugar, auxilia a identificar as barreiras à implementação da GC, e, finalmente, o último aspecto tem como característica contribuir para o planejamento de curto e longo prazo.

As perspectivas que os modelos apresentam, em regra, são: sequencial, linear, determinista e invariável do desenvolvimento da GC, que apesar de serem fundamentadas para definição de produto, não são classificadas como boa para a definição de desenvolvimento organizacional (ESCRIVÃO; SILVA, 2012). Desta forma, os modelos propostos apresentam dificuldade relativa para provar a existência de uma única sequência de etapas e estágios, boa parte dos modelos condicionam esta definição da maturidade ao 
último nível, o que pode não ser adotado pela organização pelos altos custos envolvidos na etapa.

O que é relevante para a empresa é desenvolver uma metodologia que possa ser utilizada para avaliar o modelo do ponto de vista da maturidade do produto. Para isso, é necessário verificar 11 fatores críticos de sucesso, incluindo estratégia, liderança, tecnologia da informação, gestão do conhecimento, cultura, processo, recursos, business intelligence, segurança, cliente social e avaliação (LAK; REZAEENOUR, 2018)

\section{METODOLOGIA}

O método empregado, neste estudo, foi a pesquisa bibliográfica para compor a revisão de literatura, considerando a seleção de artigos publicados na revista Perspectiva em Gestão \& Conhecimento, em 2017 e 2018. A primeira etapa foi verificar as palavras-chave dos artigos que mais se repetiam, cujos resultados encontrados foram: gestão do conhecimento, Inteligência Competitiva, Competência em Inteligência Competitiva, como é demonstrado na análise da frequência das palavras, apresentado na Tabela 1.

\begin{tabular}{|cc|}
\hline Palavras-chaves mais frequentes & Quantidade \\
Gestão do conhecimento & 6 \\
Competência da informação & 2 \\
Inteligência organizacional & 2 \\
\hline
\end{tabular}

Tabela 1 - Palavras-chave repetidas

Fonte: Elaborado pelos autores (2019)

Assim, foi observado que dos seis artigos nos quais aparece a palavra-chave gestão do conhecimento, em quatro, aparecem as palavras-chave competência em informação e inteligência competitiva com ao menos uma repetição. Desta forma, o ponto de partida para o início da pesquisa foram os quatro artigos que contêm ao menos duas das três palavraschave, como demonstrado na Figura 5. 


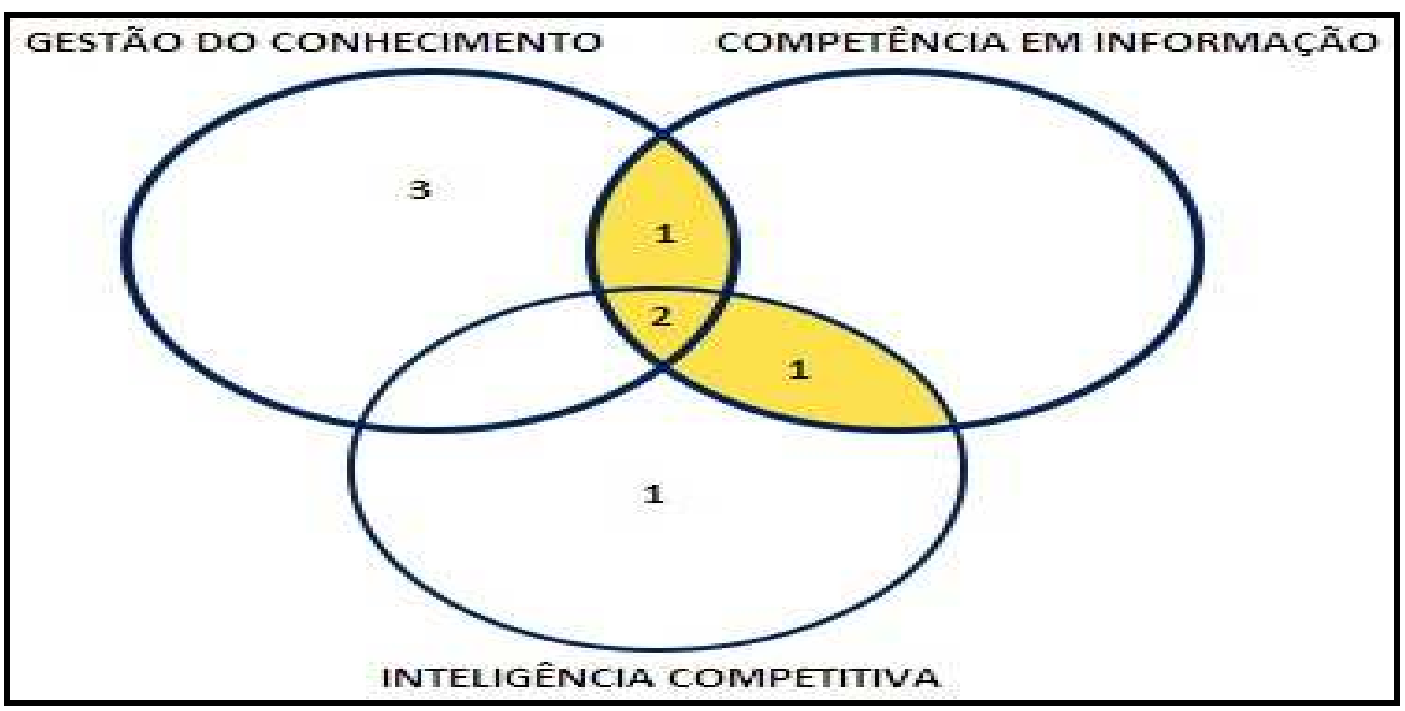

Figura 5 - Critérios para início da revisão bibliográfica Fonte: Elaborada pelos autores (2019)

O caráter bibliográfico qualitativo deste estudo tem como base os artigos selecionados na etapa inicial da pesquisa para observar os elementos que atendem ao objetivo proposto. Além dessa etapa inicial, foram analisados artigos que tratam da temática deste estudo, trazendo contribuições para a compreensão do processo que envolve a gestão do conhecimento, que é fundamental para o processo decisório nas organizações e para o desenvolvimento dos seus recursos humanos.

Na segunda etapa, os artigos foram selecionados para análise através da pesquisa exploratória na base Scopus, utilizando as palavras-chaves knowledge management, continuity, process com o operador booleano AND, resultando em 550 artigos. De forma complementar, foi necessária a aplicação de filtros para deixar apenas artigos acadêmicos em inglês e espanhol, aplicados na área de gestão e publicados em até cinco anos. $\mathrm{O}$ critério de seleção para leitura completa dos artigos foi a verificação de aderência dos títulos e resumos ao tema em questão, incluindo artigos originais e metanálises que correlacionassem os descritores citados anteriormente.

\section{ANÁLISE E DISCUSSÕES}

Segundo o IPEA, no Brasil, apenas 7\% das empresas possuíam uma área exclusivamente responsável pela GC ((BATISTA et al., 2014). Através desta lacuna, é necessário, como uma das principais ações dentro desta área, criar estratégias que busquem o alcance dos objetivos e metas e possam influenciar, de alguma forma, a gestão da empresa. Na administração pública brasileira, por exemplo, ANAC, FURNAS, BNDES e PETROBRAS apresentam uma área específica para gerir o conhecimento produzido e existente.

O início deste processo data dos anos de 1990 e não é tipicamente brasileiro, o que motiva a busca e o entendimento contínuo das experiências internacionais, que, somadas a nossa, favorecem um grande debate. Nesse sentido, os mercados têm fortalecido a gestão 
do conhecimento por meio de um aprofundamento na administração pública, onde questões como transparência no uso dos recursos públicos e avaliação de políticas públicas quanto a resultados e não somente produtos são questões novas, dando importância a participação dos interessados no decorrer do processo, ou seja, saber o que está acontecendo (BATISTA; COSTA, 2013).

Ao analisar as experiências de países que, para se profissionalizarem, institucionalizaram a gestão do conhecimento em suas estruturas de gestão pública, três países do continente americano (Canadá, México e Chile) e cinco europeus (Portugal, Reino Unido, Alemanha, Áustria e Suíça) têm experiências para servir de modelo para a administração pública brasileira com o objetivo de melhorar e contribuir com os serviços prestados pelo Estado à sociedade (BATISTA et al., 2014; MOLLEDA; MORENO; NAVARRO, 2017; SANTOS, 2016 )

A principal contribuição desses países está no tratamento de casos reais apresentados no dia a dia, de forma que já é possível ter a matéria-prima para motivar funcionários e gestores públicos encarregados de institucionalizar a GC em suas instituições. Desta forma, define-se o referencial inicial para a elaboração e implementação de políticas e estratégias de GC nas diversas estruturas, que no caso brasileiro seriam as três esferas de governo (federal, estadual e municipal) e, claro, extensivas para iniciativa privada (MASSARO; DUMAY; GARLATTI, 2015).

Assim, é importante ter um mecanismo que permita a recuperação da memória a partir dos processos de aquisição, interpretação, compartilhamento (informal e formal), memória organizacional (interna e externa) e recuperação do conhecimento (PAIS; SANTOS, 2015). Com isso, eles são considerados na organização o que já fazem e o que sabem fazer, sendo para os envolvidos de grande importância.

\subsection{MÉTODO PDCA APLICADO À GESTÃO DO CONHECIMENTO}

Com base na revisão da literatura, é possível destacar o uso de uma ferramenta de gestão, o ciclo PDCA (Plan-Do-Check-Act), proposto por Deming (2000), que, aplicado ao processo de GC, contribui para a proposição de um modelo multidisciplinar que agrega de forma simultânea as etapas organizacionais utilizadas pela área de GC, facilitando a verificação das lacunas existentes, com o objetivo de otimização de resultados, como indica a Figura 6. 


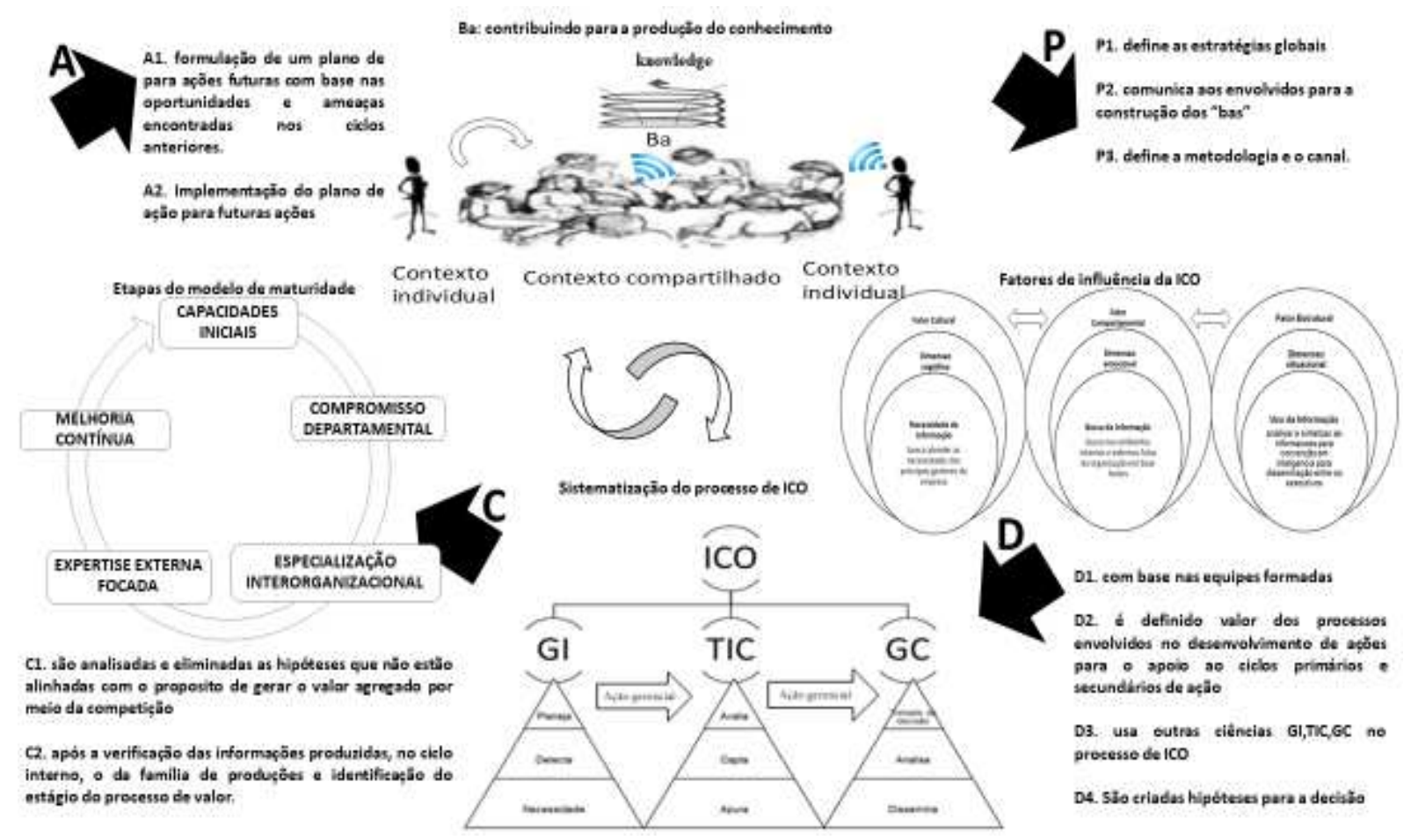

Figura 6 - Uso do PDCA para a GC Fonte: Elaborada pelos autores (2019)

Na figura 6, é possível observar que os estágios ficam bem definidos no uso do método PDCA de Deming, iniciando com a mudança do contexto de individual para compartilhado. O início do contínuo processo ocorre a partir da transformação do contexto individual em coletivo por meio de um ambiente compartilhado, onde as ideias são definidas e a metodologia de planejamento acertada com o uso dos fatores que influenciam a ICO.

A etapa da ação ou desenvolvimento é delineada nas fases de inteligência competitiva organizacional, que, organizadas em equipes, são divididas em prioridades de nível primário e secundário. Vale destacar, que influenciadas por outras ciências ou ferramentas, como a gestão da informação - Gl, tecnologia da informação e comunicação TIC e a gestão do conhecimento $-\mathrm{GC}$, facilitam a criação de hipóteses que, de maneira sistematizada, poderão ser disseminadas e escolhidas, após o recebimento dentro do fluxo de outras ações gerenciais (WANG, 2014).

Assim, com o uso das etapas do modelo de maturidade, pode-se evidenciar o desenvolvimento da alternativa escolhida e elaborar um plano de ações futuras. 0 fundamento desta ação está nas oportunidades encontradas nos ciclos anteriores, saindo de uma modelo individual para que favoreça a conversão de conhecimento tácito em explícito (BLANCO-VALBUENA et al, 2018).

É possível, também, observar que o PDCA é uma ferramenta ágil, que é validada por meio de sua eficácia para abordar as questões críticas de produção do conhecimento (GARZA-REYES et al 2018). Com o processo unificado e organizado, a organização é capaz de desenvolver uma abordagem sequencial lógica e bem definida sistematizando o fluxo produtivo. A própria aplicação de um modelo já testado, além de validar a ferramenta, 
também a dissemina para os demais gerentes, uma orientação para a busca de melhoria nos projetos vigentes e nos vindouros (GARZA-REYES, 2016).

Sendo assim, verifica-se como resposta as oportunidades do contexto organizacional a integração de quatro processos distintos e seus subprocessos em um único ciclo para melhoria contínua da produtividade operacional. De forma complementar e rápida, as soluções são encontradas entre os próprios membros da empresa que poderão, inclusive, propor a construção de indicadores já testados de eficiência e produtividade (SILVA; MEDEIROS; VIEIRA, 2017). Diante deste contexto, percebe-se o atingimento da necessidade, coleta, análise e disseminação das necessidades organizacionais, o que irá contribuir para a definição de ações especificas, para um contexto complexo e mutável, além de nortear a atividade estratégica da organização (PITELIS; WAGNER 2018). A decisão gerencial no processo decisório leva em consideração os possíveis fatores de descarte, como custo e tempo, que podem determinar que estas ações sejam classificadas como P\&D e sejam terceirizadas.

Com isso, além de reduzir o valor investido, permite que a empresa tenha como foco apenas a sua atividade-fim. Desse modo, ocorre o importante desenvolvimento do processo de conhecimento das competências organizacionais que, apoiado pelo uso do modelo de maturidade, favorece a verificação da efetividade da ação para o sucesso, em um tempo menor.

\subsection{DESENVOLVIMENTO DE COMPETÊNCIAS ORGANIZACIONAIS PARA O PROCESSO DECISÓRIO}

Como primeira etapa, indica-se o diagnóstico, por meio do uso da autoavaliação, do grau de maturidade em GC. Desta forma, o nível de maturidade serve como parâmetro utilizado para a avaliação de como a empresa gerencia o desempenho da GC (WIJETUNGE, 2017). O planejamento, por ser a próxima etapa do processo decisório, é usado para identificar as lacunas do conhecimento, e a empresa pode definir a visão, o objetivo e as estratégias de GC.

O alinhamento estratégico é importante nesta etapa, por definir a atividade da GC como um processo sistemático que visa desenvolver, criar, organizar, preservar e compartilhar o conhecimento que aumentará a eficiência dos processos, o que melhora a qualidade dos serviços prestados (YOSUA; TJAKRAATMADJA, 2015). Na sequência, é necessária a formação de equipes encarregadas de dar continuidade as atividades que devem acontecer em conjunto com a implantação da unidade central de GC para observar o atingimento dos seguintes objetivos:

- Estruturar a área de GC com uma equipe exclusiva;

- Formalizar a equipe de voluntários;

- Alocar recursos disponíveis para este processo;

- Assegurar o suporte de TI para as atividades de GC;

- Promover a cultura da GC;

- Servir como teste para outras iniciativas de GC; 
Neste contexto, a ação centra-se na incorporação do conhecimento captado através da identificação dos efeitos de múltiplos mecanismos de transferência de conhecimento, visto que nem todos os meios transferem conhecimento de forma previsível e uniforme com as melhores práticas nas normas e procedimentos relacionados com a certificação e fiscalização de processos para que os atos normativos possam ser revisados (BALLE et al., 2019; KARIMPANAL; BOUFFANAIS, 2019). Assim, são criadas comunidades de prática, que são grupos informais e interdisciplinares de pessoas unidas em torno de um interesse comum (NEELY, MARONE, 2016) que favorece o deslocamento da atenção para além dos limites organizacionais das empresas que buscam a interação com o conhecimento externo (SAVINO et al. , 2017).

As comunidades são auto organizadas a fim de permitir a colaboração de pessoas internas ou externas à organização; propiciam o veículo e o contexto para facilitar a transferência de melhores práticas por meio da identificação e discussão das características necessárias para oferecer o suporte necessário à capacidade de reutilizar modelos contingenciais com informações dependentes do contexto e das lições aprendidas (GONZÁLEZ-LOUREIRO; VILA ALONSO; SCHIUMA, 2015). Portanto, é importante criar e manter um banco de competências, que funcionará como um repositório de informações estruturadas sobre a localização do conhecimento na organização, incluindo fontes de consulta e também as pessoas ou equipes detentoras de determinado conhecimento (CAO; DUAN ; CADDEN, 2019).

\section{CONSIDERAÇÕES FINAIS}

A Gestão do Conhecimento sistematizada pela ferramenta PDCA, concretizada em ações e processos contínuos, traz bons resultados, como o envolvimento do nível estratégico da organização, que, por meio do uso da tecnologia como ferramenta, cria uma plataforma para as comunidades de práticas virtuais, o que hoje ainda não prescinde da participação dos funcionários. Desta forma, é possível investir no desenvolvimento da equipe com eventos de capacitação para os funcionários que irão aprender métodos, ferramentas e técnicas de GC, utilizando a interação grupal para gerar o conhecimento de maior impacto, gerando na própria organização um valor agregado, diferenciado em um processo de melhoria constante.

A percepção do alinhamento entre GC e objetivos organizacionais por meio da utilização de uma área dedicada ao tema, possibilita a definição, de forma clara, das competências organizacionais, que são importantes para assegurar o alinhamento das ações com os objetivos estratégicos da empresa, constituindo-se, desse modo, em um diferencial competitivo nas organizações.

Deste modo, a experiência de órgãos e empresas da administração pública revela que, apesar das dificuldades, é possível implementar com sucesso a GC, contudo, é preciso, que ocorra uma ação sistematizada por um modelo que acelere o resultado para motivar o número de organizações a participarem desta ação. 


\section{REFERÊNCIAS}

AHMAD, A.; BOSUA, R.; SCHEEPERS, R. Protecting organizational competitive advantage: A knowledge leakage perspective. Computers \& Security, v. 42, p. 27-39, 2014.

AL-EMRAN, M.; MEZHUYEV, V.; KAMALUDIN, A.; SHAALAN, K. The impact of knowledge management processes on information systems: A systematic review. International Journal of Information Management, v. 43, p. 173-187, Dec. 2018.

ALI, I.; ALI, M.; LEAL-RODRíGUEZ, A. L.; ALBORT-MORANT, G. The role of knowledge spillovers and cultural intelligence in enhancing expatriate employees' individual and team creativity. Journal of Business Research, v. 101, p. 561-573, Aug. 2019.

ASHRAFI, A.; ZARERAVASAN, A.; TRKMAN, P.; AFSHARI, S. The role of business analytics capabilities in bolstering firms' agility and performance. International Journal of Information Management, v. 47, p. 1-15, Aug. 2019.

BALLE, A. R.; STEFFEN, M. O.; CURADO, C.; OLIVEIRA, M. Interorganizational knowledge sharing in a science and technology park: the use of knowledge sharing mechanisms. Journal of Knowledge Management, 2019. Disponível em: https://www.emerald.com/insight/content/doi/10.1108/JKM-05-2018-

0328/full/pdf?title=interorganizational-knowledge-sharing-in-a-science-and-technologypark-the-use-of-knowledge-sharing-mechanisms. Acesso em: 11 jun. 2019.

BATISTA, F. F. et al. Casos reais de implantação do Modelo de Gestão do Conhecimento para Administração Pública brasileira. Brasília: IPEA, mar. 2014. (Texto para discussão; 2075)

BATISTA, F. F.; COSTA, V. S. Alinhando o modelo, o método de implementação e a prática de gestão do conhecimento (GC): o caso do Repositório do Conhecimento do Instituto de Pesquisa Econômica Aplicada (RClpea). Revista do Serviço Público, Brasília: ENAP, v. 64, n. 1, p. 59-76, jan./mar. 2013.

BEKER, K.; KIM, J.; VAN BOEKEL, M.; VAN DENBROEK, P.; KENDEOU, P. Refutation texts enhance spontaneous transfer of knowledge. Contemporary Educational Psychology, v. 56, p. 67-78, 2019.

BLANCO-VALBUENA, C. E.; BERNAL, C. Industrias Creativas y Culturales: Estudio desde el Enfoque de laGestióndelConocimiento. Información tecnológica, v. 29, n. 3, p. 15-28, 2018.

BOŽIČ, K.; DIMOVSKI, V. Business intelligence and analytics for value creation: The role of absorptive capacity. International Journal of Information Management, v. 46, p. 93-103, 2019. 
CAO, G.; DUAN, Y.; CADDEN, T. The link between information processing capability and competitive advantage mediated through decision-making effectiveness. International Journal of Information Management, v. 44, p. 121-131, 2019.

CEPEDA-CARRION, I.; MARTELO-LANDROGUEZ, S.; LEAL-RODRÍGUEZ, A. L.; LEAL-MILLÁN, A. Critical processes of knowledge management: An approach toward the creation of customer value. European Research on Management and Business Economics, v. 23, n. 1, p. 1-7, 2017.

CHEN, C. T.; HSINHUI, H.; KING, B. Shaping the organizational citizenship behavior or workplace deviance: Key determining factors in the hospitality workforce. Journal of Hospitality and Tourism Management, v. 35, p. 1-8, 2018.

CHIU, C.-N.; YANG, C.-L. Competitive advantage and simultaneous mutual influences between information technology adoption and service innovation: Moderating effects of environmental factors. Structural Change and Economic Dynamics, v. 49, p. 192-205, June 2019.

CHOO, C. W. A organização do conhecimento: como as organizações usam a informação para criar significado, construir conhecimento e tomar decisões. 2.ed. São Paulo: Ed. SENAC, 2006.

DALKIR, K. Knowledge management in theory and practice. Taylor and Francis, 2013. 1-356.

DEMING, W. E. The New Economics, for Industry, Government, Education. 2nd ed. Cambridge, MA: MIT Press, 2000

DERVIN, B. From the mind's eye of the user: the Sense-Making qualitative-quantitative methodology. In: GLAZIER, J. D.; POWELL, R.R. (Orgs.). Qualitative Research in Information Management. Englewood: Libraries Unlimited, 1992. p.61-84.

DRUCKER, P. F. The Age of Discontinuity: Guidelines to Our Changing Society. New York: Harper and Row, 1968.

EREVELLES, S.; FUKAWA, N.; SWAYNE, L. Big Data consumer analytics and the transformation of marketing.Journal of Business Research, v. 69, n. 2, p. 897-904, 2016.

ESCRIVÃO, G.; SILVA, S. L. Knowledge management maturity: Critical reflections and identification of gaps. In: 13th EUROPEAN CONFERENCE ON KNOWLEDGE MANAGEMENT, $13^{\text {th }}, 2012$, Cartagena. Proceedings ... Cartagena, 2012. p.1542-1552.

GARZA-REYES, J. A.; AL-BALUSHI, M.; ANTONY, J.; KUMAR, V. A Lean Six Sigma framework for the reduction of ship loading commercial time in the iron ore pelletising industry. Production Planning \& Control, v. 27, n. 13, p. 1092-1111, 2016. 
GARZA-REYES, J. A.; ROMERO, J. T.; GOVINDAN, K.; CHERRAFI, A.; RAMANATHAN, U. A PDCAbased approach to environmental value stream mapping (E-VSM). Journal of Cleaner Production, v. 180, p. 335-348, 2018.

GAVIRIA-MARIN, M.; MERIGÓ, J. M.; BAIER-FUENTES, H. Knowledge management: a global examination based on bibliometric analysis. Technological Forecasting and Social Change, v. 140 (C), p. 194-220, 2018.

GONZÁLEZ-LOUREIRO, M.; VILA ALONSO, M.; SCHIUMA, G. Knowledge and sustained competitive advantage: How do services firms compete? Investigacioneseuropeas de dirección y economía de la empresa, v. 21, n. 2, p. 55-57, 2015.

GREWATSCH, S.; KLEINDIENST, I. How organizational cognitive frames affect organizational capabilities: The context of corporate sustainability. Long Range Planning, v. 51, n. 4, p. 607624, 2018.

HELLE, A. C.; DESHONG, H. L.; LENGEL, G. J.; MEYER, N. A.; BUTLER, J.; MULLINS-SWEATT, S. N. Utilizing Five Factor Model facets to conceptualize counter productive, un ethical, and organizational citizenship workplace behaviors. Personality and Individual Differences, v. 135, p. 113-120, 2018.

JAKLIČ, J.; GRUBLEŠIČ, T.; POPOVIČ, A. The role of compatibility in predicting business intelligence and analytics use intentions. International Journal of Information Management, v. 43, p. 305-318, 2018.

JORGE, C. F. B.; SUTTON, M. J. D. Games como estratégia na construção e gestão do conhecimento no contexto da inteligência organizacional. Perspectivas em Gestão \& Conhecimento, v. 6, n. 1, p. 103-118, 2016.

KARIMPANAL, T. G.; BOUFFANAIS, R. Self-organizing maps for storage and transfer of knowledge in reinforcement learning. Adaptive Behavior, v. 27, n. 2, p. 111-126, 2019.

KÄRKKÄINEN, H., MYLLÄRNIEMI, J., OKKONEN, J.; SILVENTOINEN, A. Maturity assessment for implementing and using product lifecycle management in project-oriented engineering companies. International Journal of Electronic Business, v. 11, n. 2, p. 176-198, 2014.

KHADDAJ, M.; SROUR, I. Using BIM to retrofit existing buildings.Procedia Eng., 145, p.15261533, 2016.

KHAN, M. H. Knowledge, skills and organizational capabilities for structural transformation. Structural Change and Economic Dynamics, v. 48, p. 42-52, 2019.

KOOMEN, B. Set Based PLM Implementation, a Modular Approach to PLM Process Knowledge, Management and Automation. In: IFIP INTERNATIONAL CONFERENCE ON 
PRODUCT LIFECYCLE MANAGEMENT, 14 ${ }^{\text {th }}, 2017$, Seville. Proceedings ... Seville, Spain: University of Seville, 2017. p. 3-12.

KRAEMER, R.; FREIRE, P. S.; SOUZA, J. A.; DANDOLINI, G. A. Maturidade de gestão do conhecimento: uma revisão sistemática da literatura para apoiar o desenvolvimento de novos modelos de avaliação. Perspectivas em Gestão \& Conhecimento, v. 7, n. esp., p. 6679, 2017.

LAK, B.; REZAEENOUR, J. Maturity assessment of social customer knowledge management (SCKM) using fuzzy expert system. Journal of Business Economics and Management, v. 19, n. 1, p. 192-212, 2018.

LOON, M. Knowledge management practice system: theorising from an international metastandard. Journal of Business Research, v. 94, p. 432-441, 2019.

MARCHIORI, D.; FRANCO, M. Knowledge transfer in the context of inter-organizational networks: Foundations and intellectual structures. Journal of Innovation \& Knowledge, 2019. Article in Press.

MASSARO, M.; DUMAY, J.; GARLATTI, A. Public sector knowledge management: a structured literature review. Journal of Knowledge Management, v. 19, n. 3, p. 530-558, 2015.

MILLER, J. P. O milênio da inteligência competitiva. Porto Alegre: Bookman, 2002.

MOLLEDA, J. C.; MORENO, A.; NAVARRO, C. Professionalization of public relations in Latin America: A longitudinal comparative study. Public Relations Review, v. 43, n. 5, p. 10841093, 2017.

MYRODIA, A.; RANDRUP, T.; HVAM, L. Configuration lifecycle management maturity model. Computers in Industry, v. 106, p. 30-47, 2019.

NEELY, A. D.; MARONE, V. Learning in parking lots: Affinity spaces as a framework for understanding knowledge construction in informal settings. Learning, culture and social interaction, v. 11, p. 58-65, 2016.

NONAKA, I.; TOYAMA, R.; KONNO, N. SECl, Ba and Leadership: unified model of dynamic. knowledge creation. Long Range Planning, v. 33, p. 5-34, 2000.

OLAISEN, J.; REVANG, O. Exploring the performance of tacit knowledge: How to make ordinary people deliver extraordinary results in teams. International Journal of Information Management, v. 43, p. 295-304, 2018.

OLIVEIRA, R. R.; SAUER, A. B. S. Compartilhamento de conhecimento em uma empresa familiar: Um estudo à luz da investigação apreciativa. Perspectivas em Gestão \& Conhecimento, v. 6, n. 1, p. 175-199, 2016. 
PAIS, L.; SANTOS, N. R. Knowledge-sharing, cooperation and personal development. In: KRAIGER, K. et al. (Ed.). The Wiley-Blackwell handbook of the psychology of training, development and performance improvement. Oxford: Wiley-Blackwell, 2015. p. 278-302. PARK, J.; GABBARD, J. L. Factors that affect scientists' knowledge sharing behavior in health and life sciences research communities: differences between explicit and implicit knowledge. Computers in Human Behavior, v. 78, p. 326-335, 2018.

PIÇARRA, N.; GIGER, J.-C. Predicting intention to work with social robots at anticipation stage: Assessing the role of behavioral desire and anticipated emotions. Computers in Human Behavior, v. 86, p. 129-146, 2018.

PITELIS, C. N.; WAGNER, J. D. Strategic Shared Leadership and Organizational Dynamic Capabilities. The Leadership Quarterly, v. 30, n. 2, p. 233-242, 2018.

SANTOS, J. M. dos. Roots of public relations in Portugal: Changing an old paradigm. Public Relations Review, v. 42, n. 5, p. 792-800, 2016.

SANTOS, V. C. B. dos; SANTOS, C. A. dos; BELLUZZO, R. C. B. A competência em informação em articulação com a inteligência competitiva no apoio ao alinhamento estratégico das informações nas organizações. Perspectivas em Gestão \& Conhecimento, v. 6, n. 1, p. 45-60, 2016.

SAVINO, T.; MESSENI PETRUZZELLI, A.; ALBINO, V. Search and recombination process to innovate: a review of the empirical evidence and a research agenda. International Journal of Management Reviews, v. 19, n. 1, p. 54-75, 2017.

SERGEEVA, A.; ANDREEVA, T. Knowledge sharing research: Bringing context back in. Journalof Management Inquiry, v. 25, n. 3, p. 240-261, 2016.

SILVA, A. S.; MEDEIROS, C. F.; VIEIRA, R. K. Cleaner Production and PDCA cycle: Practical application for reducing the Cans Loss Index in a beverage company. Journal of cleaner production, v. 150, p. 324-338, 2017.

SIMANCA, M. M.; MONTOYA, L. A.; BERNAL, C. A. Gestión del conocimiento en cadenas productivas: El caso de lacadena láctea en Colombia. Información tecnológica, v. 27, n. 3, p. 93-106, 2016.

SIMEONE, L.; SECUNDO, G.; SCHIUMA, G. Art sand design as translational mechanisms for academic entrepreneurship: The metaLABat Harvard case study. Journal of Business Research, v. 85, p. 434-443, 2018.

ŠTEFÁNIKOVÁ, L'.; MASÀROVÀ, G. The need of complex competitive intelligence. ProcediaSocial and Behavioral Sciences, v. 110, p. 669-677, 2014. 
TEIXEIRA, T. M. C.; VALENTIM, M. L. P. Inteligência competitiva organizacional: um estudo teórico. Perspectivas em Gestão \& Conhecimento, v. 6, n. 1, p. 3-15, 2016.

TRIEU, V.-H. Getting value from Business Intelligence systems: A review and research agenda. Decision Support Systems, v. 93, p. 111-124, 2017.

WANG, H. -C. Distinguishing the adoption of business intelligence systems from their implementation: The role of managers' personality profiles. Behaviour \& Information Technology, v. 33, n. 10, p. 1082-1092, 2014.

WIJETUNGE, P. Assessing Knowledge Management Maturity level of a university library: a case study from Sri Lanka. Qualitative and Quantitative Methods in Libraries, v. 1, n. 3, p. 349-356, 2017.

WILLERDING, I. A. V.; KRAUSE, M. G.; LAPOLLI, E. M. Gestão de pessoas e gestão do conhecimento à luz da estética organizacional. Perspectivas em Gestão \& Conhecimento, v. 6, n. 1, p. 141-154, 2016.

YOSUA, A.; TJAKRAATMADJA, J. H. Assessment and Planning of Knowledge Management at PT Dirgantara Indonesia (Persero). Procedia-Social and Behavioral Sciences, v. 169, p. 109124, 2015.

ZHANG, L.; CHENG, J. Effect of knowledge leadership on knowledge sharing in engineering project design teams: The role of social capital. Project Management Journal, v. 46, n. 5, p. 111-124, 2015. 